\title{
ASPECTOS BIOLÓGICOS DA TRAÇA DO TOMATEIRO E ARMAZENAMENTO DE SEUS OVOS E PUPAS A BAIXAS TEMPERATURAS
}

\author{
Laurici Maria Pires ${ }^{1}$ \\ Irene M.R. Marques ${ }^{2}$
}

\section{RESUMO}

Os dados biológicos de Tuta absoluta (Meyrick) foram obtidos em laboratório, onde a temperatura e umidade oscilaram entre 24,5 a $31,2^{\circ} \mathrm{C}$ e 45 a $100 \%$ de U.R. sendo registrado uma média de $28^{\circ} \mathrm{C}$ e $75 \%$ U.R., utilizando-se como hospedeiro natural a espécie Lycopersicum sculentum var. IPA5. Os períodos de incubação, larval e pupal, foram respectivamente $4,6 \pm 0,05 ; 11,9 \pm 0,15$ e $6,5 \pm 0,33$ dias. O período de ovo até a emergência do adulto foi de $22,2 \pm 0,35$ dias. Buscou-se o aprimoramento da metodologia de criação de $T$. absoluta, mediante o armazenamento de ovos e pupas em geladeira $\left(10 \pm 1^{\circ} \mathrm{C}\right)$. Após os períodos de armazenamento os tratamentos foram retirados da geladeira e mantidos à temperatura ambiente até a eclosão das lagartas e emergência de adultos, sendo avaliadas suas viabilidades. O armazenamento de ovos e pupas de T. absoluta em geladeira permitiu o retardamento do desenvolvimento das respectivas formas biológicas em até 15 dias, sem perdas significativas de viabilidades das lagartas e adultos correspondentes.

Palavras-chave: Inseto, biologia, traça-do-tomateiro, armazenamento de ovos e pupas, Tuta absoluta.

${ }^{1}$ UFRPE, Departamento de Agronomia, Rua Dom Manoel de Medeiros, s/n, 52171900, Recife-PE.

${ }^{2}$ UFPE, Departamento de Zoologia, Av. Prof. M Rego, 1235 Cd. Universitária, 50670 420 Recife-PE. 


\section{ABSTRACT \\ BIOLOGICAL DATA OF THE TOMATO PINWORM AND STORAGE OF ITS EGGS AND PUPAE AT LOW TEMPERATURE}

The biological data of Tuta absoluta (Meyrick) were obtained at laboratory conditions, where temperature and humidity between 24,5 $31,2^{\circ} \mathrm{C}$ and $45-100 \%$ R.H., by average of $28^{\circ} \mathrm{C}$ and $75 \%$ R.U., using as natural host the species Lycopersicum esculentum var. IPA5. The incubation, larval and pupal periods were respectively 4,6 $40,05,11,9 \pm$ 0,15 and $6,5 \pm 0,33$ days. The period from egg to adult emergence was $22,2 \pm 0,35$ days. The methodology was improved by storing the eggs and pupae in a refrigerator $\left(10 \pm 1^{\circ} \mathrm{C}\right)$, during different periods. After storage, the materials from different treatments were removed from the refrigerator and maintained under laboratory conditions until the egg hatching and adult emergence. The storage of the eggs and pupae in a refrigerator allowed a delay of up to 15 days of the respective biological stages, without significative loss of viability of corresponding larvae and adults.

Key words: Insecta, biology, pinworm, storage of eggs and pupae, Tuta absoluta.

\section{INTRODUÇÃO}

A traça Tuta absoluta (Meyrick) (Lepidoptera, Gelechiidae), constitui um dos grandes problemas entomológicos da cultura do tomateiro. As plantas severamente atacadas ficam totalmente danificadas e os prejuízos são expressivos principalmente considerando-se o alto custo do investimento desta cultura (Nakano \& Paulo, 1983).

Apesar dos resultados de pesquisas, T. absoluta ainda continua sendo uma praga séria que causa anualmente prejuízos consideráveis. $\mathrm{O}$ desenvolvimento de pesquisas com esse inseto defronta-se com dificuldades que impedem o rápido progresso, entre as quais, a manutenção da sua criação em laboratório, uma vez que se desenvolve apenas em dieta 
natural, o tomateiro, reconhecidamente uma cultura difícil de ser conduzida sem a utilização de produtos agroquímicos. Além disso, o comportamento reprodutivo desta praga leva à posturas concentradas num curto período, que em nossas condições ambientais passam rapidamente à próxima fase de desenvolvimento, dificultando o controle sobre o estádio que se deseja utilizar nos experimentos. Assim sendo, a elaboração desta pesquisa visou a ampliação do período de ovo e pupa, através da conservação em baixas temperaturas. Harris et al. (1984) discorreram sobre a importância de se desenvolver uma técnica que possa retardar o desenvolvimento do inseto para que seja usado em momento oportuno, facilitando diversos estudos.

Embora não se encontre na literatura referências sobre o armazenamento de ovos e pupas de T. absoluta, trabalhos envolvendo outras pragas e/ou parasitóides, foram realizados obtendo-se resultados satisfatórios (Hussey \& Gostick 1964, Salmeron et al 1985, Parra et al 1971, Stinner et al 1974, Ho 1979).

Neste contexto, pesquisou-se o aprimoramento da metodologia de criação de T. absoluta, mediante armazenamento de ovos e pupas em geladeira, permitindo maior racionalização da criação e a utilização dessas formas biológicas em tempo apropriado para a realização dos experimentos.

\section{MATERIAL E MÉTODOS}

O trabalho foi desenvolvido no Laboratório de Controle Biológico da Área de Fitossanidade do Departamento de Agronomia da Universidade Federal Rural de Pernambuco.

A criação de T. absoluta foi iniciada a partir de pupas fornecidas pelo Setor de Entomologia do CPATSA - EMBRAPA, Petrolina, PE. Após a emergência, os adultos foram liberados em uma gaiola telada $(1,0$ x $1,0 \times 0,5 \mathrm{~m}$ ) e alimentados com solução açucarada a $10 \%$, para a obtenção de posturas sobre folhas de tomateiro var. IPA5.

Os aspectos biológicos de T. absoluta observados foram: período de incubação de ovos, período larval e pupal. Para tanto, foram individualizados folíolos de tomateiro contendo cerca de 15 ovos, com idade de $24 \mathrm{~h}$, em pequenas caixas de acrílico forradas com papel de filtro. Os 
folíolos danificados pelas lagartas foram substituídos periodicamente por folíolos novos. O experimento foi realizado em laboratório, onde a temperatura e umidade oscilaram entre 24,5 a $31,2^{\circ} \mathrm{C}$ e 45 a $100 \%$ de U.R. sendo registrado uma média de $28^{\circ} \mathrm{C}$ e $75 \%$ U.R.

Para o armazenamento de ovos de T. absoluta em geladeira $\left(10 \pm 1^{\circ} \mathrm{C}\right)$ utilizou-se segmentos de galhos infestados com aproximadamente 20 ovos com idade de $24 \mathrm{~h}$, individualizados em caixas de acrílico, para cada repetição. $O$ experimento constituiu de cinco intervalos de armazenamento $(0,5,10,15$ e 20 dias $)$ e quatro repetições. Após os períodos de armazenamento os tratamentos foram retirados da geladeira e mantidos à temperatura ambiente, sendo registrado uma média de $29^{\circ} \mathrm{C}$ (máx de 30,8 e min de $26,3^{\circ} \mathrm{C}$ ) e $79 \%$ U.R., até a eclosão e desenvolvimento das lagartas.

O experimento com o armazenamento de pupas em geladeira $\left(10 \pm 1^{\circ} \mathrm{C}\right)$, constou também de cinco intervalos de armazenamento $(0,5$, 10,15 e 20 dias de armazenamento) e 30 repetições. Cada repetição constou de pupas recém formadas, obtidas da criação e individualizadas em pequenos tubos de vidro de fundo chato com $8,5 \mathrm{~cm}$ de altura por $2 \mathrm{~cm}$ de diâmetro vedados por um tecido tipo "voil". Após os períodos de armazenamento as pupas foram retiradas da geladeira e mantidas à temperatura ambiente média de $27^{\circ} \mathrm{C}$ (máx de 30 e min de $26^{\circ} \mathrm{C}$ ), para observação do número de adultos emergidos, registrando-se a presença de deformações.

Para os dados de biologia obtidos foram calculadas as médias aritméticas, intervalos de confiança, coeficiente de variação e porcentagens de viabilidade. Os experimentos seguiram um delineamento experimental inteiramente casualizado e para fins de análise estatística os dados foram transformados em arco seno $\sqrt{x} / 100$, para a porcentagem de lagartas que penetraram nas folhas e $\sqrt{ } \mathrm{x}+1$ para viabilidade pupal, deformação de adultos e período pupal após o armazenamento. A comparação entre as médias dos tratamentos foi realizada através do teste de Tukey $(\mathrm{P} \leq 0,05)$.

\section{RESULTADOS E DISCUSSÃO}

Embora a biologia de T. absoluta seja bastante explorada, o conhecimento de alguns aspectos biológicos deste inseto se fizeram neces- 
sários novos trabalhos nas condições em que se desenvolveu esta pesquisa, uma vez que foi utilizada a variedade regional de tomate IPA5. Giustolin e Vendramin (1994) observaram que a fonte de alimento, a temperatura e a umidade relativa influenciaram na duração das fases de desenvolvimento de $T$. absoluta, devendo estes aspectos ser observados para cada condição.

\section{Biologia}

O período de incubação de ovos variou de 4 a 6 dias, com média de 4,6 dias e viabilidade de $87,1 \%$ (Tabela 1 ), havendo uma maior frequiência de eclosão das lagartas no $5^{\circ}$ dia. O período larval teve duração média de 11,9 dias, com variação de 9 a 14 dias. A viabilidade do estágio larval foi de $24,7 \%$. Dados semelhantes foram observados por Haji et al. (1988) que obtiveram viabilidade larval de 20,9\%. Da mesma forma, Coelho e França (1987) registraram alta mortalidade após a eclosão. A fase de pupa teve duração média de 6,5 dias, com um período mínimo 4 e máximo de 10 dias e viabilidade de $71,4 \%$. A duração do período de ovo até a emergência do adulto foi de 22,2 dias, com um período 19 a 26 dias. Estas observações estão de acordo com Coelho e França (1987) e Haji et al. (1988).

Tabela 1. Dados biológicos de Tuta absoluta, obtidos em condições de laboratório $\left(28^{\circ} \mathrm{C}\right.$ e $75 \%$ U.R.).

\begin{tabular}{cccccc}
\hline \multicolumn{5}{c}{ DURAÇÃO EM DIAS } \\
\hline & Min & Max & Média & Viabilidade \% & CV \% \\
\hline Incubação de ovos & 4 & 6 & $4,6 \pm 0,05$ & 87,11 & 4,50 \\
Período larval & 9 & 14 & $11,9 \pm 0,15$ & 24,7 & 5,65 \\
$\begin{array}{c}\text { Período pupal } \\
\text { Período de ovo a } \\
\text { adulto }\end{array}$ & 4 & 10 & $6,5 \pm 0,33$ & 71,43 & 22,33 \\
\hline
\end{tabular}

\section{Armazenamento}

A eclosão das lagartas procedentes de ovos armazenados, teve início dois dias após estes serem retirados da geladeira, em todos os in- 
tervalos. Não ocorreu diferença significativa na viabilidade dos ovos entre a testemunha e os tratamentos até o décimo quinto dia de armazenamento (Tabela 2), obtendo-se inclusive porcentagens de viabilidade superiores àquela observada neste trabalho durante a obtenção dos dados biológicos de T. absoluta.

Tabela 2. Viabilidade média de ovos de Tuta absoluta armazenados em geladeira $\left(10 \pm 1^{\circ} \mathrm{C}\right)$ e porcentagem média de lagartas que penetraram nas folhas, provenientes dos ovos armazenados.

\begin{tabular}{ccc}
\hline $\begin{array}{c}\text { Intervalos de } \\
\text { armazenamento }\end{array}$ & $\begin{array}{c}\text { Viabilidade dos } \\
\text { ovos }\end{array}$ & \% de lagartas que penetraram nas folhas ${ }^{1,2}$ \\
\hline 0 & $92,5 \pm 1,44 \mathrm{a}$ & $78,4 \pm 7,89 \mathrm{a}$ \\
5 & $86,3 \pm 2,39 \mathrm{ab}$ & $77,1 \pm 9,61 \mathrm{a}$ \\
10 & $90,0 \pm 2,04 \mathrm{ab}$ & $59,5 \pm 15,32 \mathrm{a}$ \\
15 & $85,2 \pm 2,87 \mathrm{ab}$ & $56,9 \pm 4,78 \mathrm{a}$ \\
20 & $74,9 \pm 6,79 \mathrm{~b}$ & - \\
$\mathrm{CV}$ & $8,49 \%$ & $15,25 \%$ \\
\hline
\end{tabular}

'Médias seguidas de mesma letra não diferem entre si pelo teste de Tukey ao nível de $5 \%$ de probabilidade.

${ }^{2}$ Dados transformados em arco seno $\sqrt{x / 100}$.

$\mathrm{Na}$ literatura não foi encontrado dado algum sobre o armazenamento de ovos de T. absoluta. Porém Branson (1978) observou que foi possível armazenar ovos de Diabrotica virgifera a $10^{\circ} \mathrm{C}$ por 52 semanas, sem perdas da viabilidade com relação à testemunha. Schimidt (1991) trabalhando com armazenamento de ovos de Anagasta kuehniella (Zeller), a uma temperatura de $10,4^{\circ} \mathrm{C}$, não atingiu seu objetivo no prolongamento do período de incubação, observando uma redução drástica da viabilidade dos ovos armazenados por 16 dias, fato que difere dos dados obtidos neste trabalho para T. absoluta, que apresentou uma viabi- 
lidade de $85,2 \%$ para os ovos armazenados por 15 dias, naquela temperatura.

Após o décimo dia de armazenamento, observou-se uma redução gradual na viabilidade dos ovos de T. absoluta, com o aumento do período de estocagem, embora estes valores tenham permanecido elevados, não ultrapassando o limite inferior a 74,9\% (Tabela 5).

Houve diferença significativa entre a testemunha e o tratamento com 20 dias de armazenamento, embora este não tenha diferido dos demais (Tabela 2). Este fato indica que, apesar de ter diferido estatisticamente da testemunha, o armazenamento de ovos de $T$. absoluta em geladeira por 20 dias, pode ser considerado viável, uma vez que $74,9 \%$, é uma taxa relativamente alta, que garante descendência suficiente para se manter a criação do inseto.

Com relação ao desenvolvimento das lagartas de $T$. absoluta após a ećlosão, observou-se que a porcentagem que conseguiu penetrar nas folhas de tomateiro iniciando a alimentação e conseqüente desenvolvimento, decresceu a medida que aumentou o período de armazenamento (Tabela 6), embora essa diferença não tenha sido significativa estatisticamente (Tabela 2). Resultados obtidos neste trabalho em relação à biologia desta espécie mostram que a viabilidade larval de T. absoluta em condições de laboratório é baixa, ocorrendo uma alta mortalidade logo após a eclosão. Conforme foi observado, os níveis de viabilidade larval poderão ser mantidos elevados, caso as lagartas possam ser mantidas em uma pequena muda de tomateiro, onde as folhas permaneçam tenras por mais tempo, proporcionando uma maior fonte de alimento, em condições mais arejadas, uma vez que o acompanhamento do desenvolvimento das lagartas em laboratório para os trabalhos de biologia e armazenamento de ovos é realizado em placas de Petri, utilizando-se apenas um pedaço de tecido vegetal como fonte de alimento.

As pupas armazenadas por cinco dias apresentaram viabilidade de $96,7 \%$ com apenas $6,9 \%$ de deformação dos adultos emergidos, valores idênticos àqueles observados para a testemunha, o que indica ser este um período bastante favorável para armazenamento (Tabela 3). Aos 15 dias de armazenamento a viabilidade pupal foi de $90 \%$, não diferindo 
Tabela 3. Viabilidade de pupas de Tuta absoluta armazenadas em geladeira $\left(10 \pm^{\circ} \mathrm{C}\right), \%$ de deformação de adultos e período pupal após o armazenamento.

\begin{tabular}{cccc}
\hline $\begin{array}{c}\text { Período de armazenamento } \\
\text { (dias) }\end{array}$ & $\begin{array}{c}\text { Viabilidade pupal } \\
(\%)\end{array}$ & $\begin{array}{c}\text { Deformação de adultos }^{1} \\
(\%)\end{array}$ & $\begin{array}{c}\text { Período pupal }^{1} \\
\text { (dias) }\end{array}$ \\
\hline 0 & $96,7 \mathrm{a}$ & $6,9 \mathrm{a}$ & $5,6 \pm 0,19 \mathrm{ab}$ \\
$(6-8)$ \\
5 & $96,7 \mathrm{a}$ & $6,9 \mathrm{a}$ & $\begin{array}{c}4,9 \pm 0,21 \mathrm{bc} \\
(2-6)\end{array}$ \\
10 & $83,3 \mathrm{ab}$ & $28,0 \mathrm{ab}$ & $4,5 \pm 0,13 \mathrm{~cd}$ \\
& $90,0 \mathrm{ab}$ & $29,6 \mathrm{ab}$ & $5,7 \pm 0,18 \mathrm{a}$ \\
15 & $70,0 \mathrm{~b}$ & $47,6 \mathrm{~b}$ & $3,8 \pm 0,33 \mathrm{~d}$ \\
& & $1-7)$ \\
\hline 20 & $9,82 \%$ & $14,97 \%$ & $9,53 \%$ \\
\hline $\mathrm{CV}$ & & &
\end{tabular}

${ }^{1}$ Médias seguidas de mesma letra não diferem entre si pelo teste de Tukey ao nível de $5 \%$ de probabilidade. Dados transformados para $\sqrt{x+1}$.

Tabela 4. Frequiência de eclosão de lagartas de Tuta absoluta em condições de laboratório $\left(28^{\circ} \mathrm{C}\right.$ e $75 \%$ U.R.).

\begin{tabular}{cc}
\hline Período de incubação (dias) & Eclosão de lagartas (\%) \\
\hline 4 & 31,6 \\
5 & 53,8 \\
6 & 1,8 \\
\hline
\end{tabular}

Tabela 5. Viabilidade de ovos de Tuta absoluta armazenados em geladeira $\left(10 \pm 1^{\circ} \mathrm{C}\right)$, por diferentes períodos.

\begin{tabular}{cc}
\hline Dias de armazenamento & Viabilidade de ovos (\%) \\
\hline 0 & 92,5 \\
5 & 86,3 \\
10 & 90,9 \\
15 & 85,2 \\
20 & 74,9 \\
\hline
\end{tabular}


estatisticamente do tratamento de 10 dias que foi $83,3 \%$. Aos 20 dias de armazenamento foi possível obter uma viabilidade pupal de $70 \%$, que embora tenha diferido estatisticamente da testemunha, não diferiu dos tratamentos de 10 e 15 dias, permitindo ainda a obtenção de um razoável nível de emergência de adultos. Na literatura consultada, apenas Parra $e t$ al. (1971) mostraram que foi possível conservar pupas de Spodoptera frugiperda por 20 dias a $10^{\circ} \mathrm{C}$, obtendo-se uma viabilidade de $90 \%$.

Com relação à deformação de adultos provenientes das pupas armazenadas, a porcentagem aumentou proporcionalmente com o aumento do período de armazenamento, ocorrendo diferenças significativas apenas entre a testemunha e o tratamento de 20 dias, embora este não tenha diferido estatisticamente dos tratamentos de 10 e 15 dias (Tabela 7).

Assim como ocorre com as fases de ovo e lagarta, em criação à base de dieta natural, uma grande quantidade de pupas é formada em pouco espaço de tempo, surgindo a necessidade de se prolongar este estágio de desenvolvimento com o objetivo de retardar a emergência dos adultos e consequentemente, posterior oviposição. Esse fato foi relevante neste trabalho quando das reduções drásticas dos tomateiros, fonte de alimento da praga, devido a ação de doenças e pragas.

Ao serem transferidas da geladeira para a temperatura ambiente, as pupas apresentaram períodos diferentes até a emergência dos adultos (Tabela 3). De acordo com os resultados obtidos, a média em dias para a emergência dos adultos de T. absoluta, após a retirada das pupas da geladeira, mostrou uma tendência a diminuir à medida que aumentou o período de armazenamento, exceto para o tratamento de 15 dias (Tabela 8). Este fato demonstra que $10^{\circ} \mathrm{C}$, ainda é uma temperatura muito elevada para afetar o metabolismo de substâncias vitais que interrompam o desenvolvimento do inseto. Fridller et al. (1988) citado por Schimidt (1991), relataram que, em relação ąos insetos, até o limite de $-150^{\circ} \mathrm{C}$ ocorrem reações químicas, embora com velocidade reduzida. $\mathrm{O}$ prolongamento do período pupal apresentado no tratamento de 15 dias, por ocasião da retirada das pupas da geladeira a $25^{\circ} \mathrm{C}$, provavelmente se deu devido à queda de temperatura ambiente, abaixo da média registrada durante o experimento. 
Tabela 6. Porcentagem de lagartas de Tuta absoluta, provenientes de ovos armazenados em geladeira $\left(10 \pm 1^{\circ} \mathrm{C}\right)$, que penetraram em folhas de tomateiro.

Período de armazenamento (dias) Lagartas (\%)

\begin{tabular}{cc}
\hline 0 & 78,4 \\
5 & 77,1 \\
10 & 59,5 \\
15 & 56,9 \\
\hline
\end{tabular}

Tabela 7. Viabilidade de pupas e deformação de adultos de Tuta absoluta armazenadas em geladeira $\left(10 \pm 1^{\circ} \mathrm{C}\right)$.

\begin{tabular}{ccc}
\hline $\begin{array}{c}\text { Período de armazenamento } \\
\text { (dias) }\end{array}$ & $\begin{array}{c}\text { Viabilidade } \\
\text { Pupal(\%) }\end{array}$ & $\begin{array}{c}\text { Deformidade } \\
\text { de adultos(\%) }\end{array}$ \\
\hline 0 & 96,7 & 6,9 \\
5 & 96,7 & 6,9 \\
10 & 83,3 & 28,0 \\
15 & 90,0 & 29,6 \\
20 & 70,0 & 47,6 \\
\hline
\end{tabular}

Tabela 8. Período pupal de Tuta absoluta após armazenamento em geladeira $\left(10 \pm 1^{\circ} \mathrm{C}\right)$

\begin{tabular}{cc}
\hline Período de armazenamento (dias) & Período pupal (dias) \\
\hline Test & 5,6 \\
5 & 4,9 \\
10 & 4,5 \\
15 & 5,7 \\
20 & 3,8 \\
\hline
\end{tabular}




\section{CONCLUSÃO}

Considerando-se os resultados de armazenamento de ovos e pupas de T. absoluta a $10 \pm 1^{\circ} \mathrm{C}$, observou-se que, até 15 dias, é viável o retardamento do período de incubação de ovos e prolongamento do período pupal, sem que haja efeito significativo sobre suas viabilidades, beneficiando o desenvolvimento de pesquisas sobre T. absoluta, que dependem da regulação da eclosão de lagartas e emergência de adultos.

\section{REFERÊNCIAS BIBLIOGRÁFICAS}

BRANSON, T.F., 1978. Optimum Temperature for Long-Term Storage of Eggs of Diabrotica virgifera (Coleoptera : Crysomelidae). Entomol. Exp. Appl., 24:199-200.

COELHO, M. C. F. \& F. H. FRANÇA, 1987. Biologia, Quetotaxia da Larva e Descrição do Adulto da Traça-do-Tomateiro. Pesq. Agropec. Bras., 22(2):129-135.

GIUSTOLIM, T.A. \& J.D. VENDRAMIM, 1994. Efeito de Duas Espécies de Tomateiro na Biologia de Scrobipalpuloides absoluta (Meyrick). An. Soc. Entomol., 23(3):511-517.

HAJI, F.N.P.; J.R.P. PARRA; J.P. SILVA \& J.G.S. BATISTA, 1988. Biologia da Traça do Tomateiro sob Condições de Laboratório. Pesq. Agropec. Bras., 23(2): 107-110.

HARRIS, C.R.; J.W. WHISTLECRAFT; H.J. SVEC; J.H. TOLMAN \& A.D. TOMLIN, 1984. Outdoor Rearing Technique for Mass Production of Onion Maggots (Diptera: Anthomyiidae). Journ. Econ. Entom., 77(3):824-827.

HO, K., 1979. Studies on the Effect of Low Temperature Storage of Apanteles plutellae. Phytopathol. Entomol., 6:16-22.

HUSSEY, W. \& K.G. GOSTICK, 1964. Effects of Low - Temperature Storage on the Eggs of Spodoptera littoralis (Boisduval). Nature, 49(42):323-324.

NAKANO, O. \& A.D.PAULO, 1983. As Traças do Tomateiro. Agroquímica, 2:8-12. 
PARRA, J.R.P.; P.R. REIS \& A.H. CAMARGO, 1971. Resistência de Milho à Spodoptera frugiperda ( J.E. Smith ). II - Efeito de Diferentes Temperaturas Sobre a Conservação de Pupas. Rev. Agric., 46(1):32-40.

SALMERON, E.; F.M. WIENDL \& J.M.M. WALDER, 1985. Influência da Temperatura na Manutenção de Ovos de Diatraea saccharalis (F.) ( Lep., Pyralidae) para Programas de Criação Massal. Rev. Agric., 61(1):16-25.

SCHMIDT, F.G.V., 1991. Armazenamento em Baixas Temperaturas, de Ovos de Anagasta kuehniella (Zeller, 1879) e de Corcyra cephalonica (Stainton, 1865) Visando a Produção de Trichogramma spp. Piracicaba, 108p. Dissertação (Mestrado) - Escola Superior de Agricultura "Luiz de Queiroz"/USP.

STINNER, R.E.; R.L. RIDGWAY \& R.E. KINZER, 1974. Storage, Manipulation of Emergence, and Estimation of Numbers of Trichogramma pretiosum. Environ. Entomol., 3(3)505-507. 